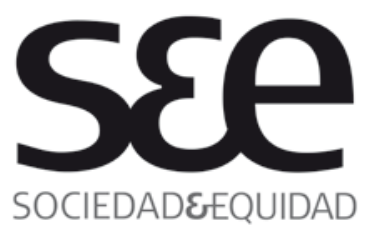

\title{
Barreras interaccionales en la atención materno-infantil a inmigrantes peruanas
}

\author{
Interactional barriers in mother and child \\ health service for Peruvian immigrants
}

\begin{tabular}{|c|c|}
\hline Nombre: & $\begin{array}{l}\text { Camila Scozia Leighton }{ }^{(1)} \\
\text { Catalina Leiva Báez } \\
\text { Natalia Garrido Maldonado } \\
\text { Andrea Álvarez Carimoney }\end{array}$ \\
\hline $\begin{array}{l}\text { Filiación: } \\
\text { País: }\end{array}$ & Universidad de Chile \\
\hline Contacto: & $\begin{array}{l}\text { cscozia@ug.uchile.cl } \\
\text { cjleivabaez@ug.uchile.cl } \\
\text { ngarridom@ug.uchile.cl } \\
\text { andrealcar@hotmail.com }\end{array}$ \\
\hline
\end{tabular}

\section{Resumen}

En los últimos años, la llegada de inmigrantes peruanas como usuarias de atención materno-infantil al Servicio de Salud Metropolitano Central (SSMC) ha supuesto numerosos desafíos tanto para ellas como para la población nacional. El objetivo de esta investigación es conocer las barreras de acceso a una atención de calidad que surgen para estas usuarias en las diversas interacciones entre ellas y los funcionarios del sistema de atención materno-infantil, en dos consultorios dependientes del SSMC. Utilizando un enfoque cualitativo, se

\footnotetext{
${ }^{1}$ Camila Scozia Leighton es Licenciada en Antropología, con mención en Antropología Social, Universidad de Chile. Sus principales líneas de investigación son salud, interculturalidad, racismo, discriminación y niñez migrante, en relación a la migración peruana en Chile. Catalina Leiva Báez es Licenciada en Antropología con mención en Antropología Social, Universidad de Chile. Sus principales temáticas de investigación son educación, salud, e interculturalidad en el contexto de las migraciones internacionales. Natalia Garrido Maldonado es Licenciada en Antropología con mención en Antropología Social, Universidad de Chile. Sus principales temáticas de investigación son los procesos de integración, exclusión y movilidad social y económica que experimentan los migrantes peruanos en Santiago de Chile. Andrea Álvarez Carimoney es Bachiller en Humanidades y Ciencias Sociales y Licenciada en Antropología con mención en Antropología Social, Universidad de Chile. Sus principales temáticas de investigación son antropología médica, género, sexualidad, migración, interculturalidad, medioambiente y salud.
} 


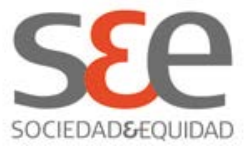

realizaron entrevistas semiestructuradas a trece inmigrantes peruanas usuarias de atención materno-infantil y trece funcionarios. Se rescata como principal resultado de la investigación el carácter emergente de las barreras, en tanto producto social efecto de las interacciones de carácter mixto entre los actores, relaciones que se nutren y articulan con otros contenidos existentes en la sociedad, como la construcción y representación del Otro y las expectativas mutuas que de allí se derivan. En este modelo relacional, los inmigrantes peruanos no se presentaron como un alter culturalmente muy distante en cuanto a sus prácticas médicas, por lo que se propone redefinir las actuales orientaciones hacia un modelo de atención que en lugar de relevar diferencias no detectadas, considere las particularidades del suj eto migrado.

\title{
Palabras Claves
}

Migración peruana, Salud Intercultural, Atención materno-infantil, Barreras de atención, Interacción, Estigma, Santiago de Chile.

\begin{abstract}
In the last years, the arrival of Peruvian immigrants as users of mother and child health care units at the Metropolitan Health Service Central has supposed many challenges for them and the national population. The aim of this investigation is to know about the barriers to a quality health service access for these users. These barriers arise from the interactions between them and the employees at mother and child health unit, in two medical centers dependent on the Metropolitan Health Service Central. Using a qualitative approach, semi structured interviews have been applied to thirteen Peruvian immigrants and thirteen employees. Main results refer to the emerging nature of these barriers as a social product of the mixed contacts between different actors, relations that are nourished and articulated with other existing contents in society, as the construction and representation of the Other, and mutual expectations. In this relational model, Peruvian immigrants didn't appear as a culturally distant group as far as medical practices are concerned; because of that we propose to re-define the current orientations towards a service model that instead of focusing on non detected differences, takes the particularities of the migrated subject into consideration.
\end{abstract}

\section{Keywords}

Peruvian migration, Intercultural Health, Mother and child health service, Access barriers, Interaction, Stigma, Santiago de Chile. 


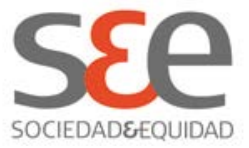

\section{Introducción}

La llegada de usuarias inmigrantes peruanas al sistema de salud público ha supuesto numerosos desafíos tanto para la población extranjera como para la nacional y se ha posicionado como un tema que merece ser analizado desde distintas perspectivas. Los equipos de salud han comenzado a cuestionar las relaciones que se establecen entre el sistema de salud y los usuarios extranjeros. La presente investigación se realizó en el marco de la cátedra de Antropología Médica de la carrera de Antropología Social en la Universidad de Chile, en base a una petición e inquietud propuesta desde el Servicio de Salud Metropolitano Central (SSMC), dependiente del Ministerio de Salud de Chile.

En particular se nos propuso investigar las razones por las cuales las usuarias peruanas comienzan sus controles gestacionales más tardíamente que las usuarias chilenas. Este fenómeno afecta negativamente el cumplimiento de las metas sanitarias de la atención materno-infantil, que apuntan a controlar la población gestante para poder realizar intervenciones oportunas enfocadas en la salud de madres y niños, por otro lado, al no cumplirse éstas, los funcionarios no reciben el incentivo económico correspondiente. Los consultorios del área del SSMC correspondiente a las comunas de Santiago, Estación Central, Pedro Aguirre Cerda, Maipú y Cerrillos, reciben recurrentemente población peruana, y son estas mujeres inmigrantes quienes no llegan a control antes del primer trimestre de embarazo.

El objetivo de esta investigación es identificar, describir y analizar las barreras de acceso a una atención de calidad que surgen para las usuarias inmigrantes peruanas en las diversas interacciones entre ellas y los funcionarios del sistema de atención materno-infantil, en dos consultorios dependientes del SSMC. Entendemos la interacción entre estos agentes como un "contacto mixto" (Goffman, 2006), es decir, una interacción donde existe presencia física inmediata de estigmatizados y estigmatizadores. Cabe puntualizar que entendemos el "acceso" como un elemento complejo. Si bien el acceso está por derecho garantizado, en la práctica significa no sólo el hecho concreto de que las potenciales usuarias se atiendan efectivamente en los centros de salud, sino además, que sea a una atención que, problematizando las variables involucradas en las interacciones establecidas entre funcionarios de la salud e inmigrantes, cumpla los objetivos del organismo sanitario, satisfaciendo a la vez las necesidades de las usuarias. En relación a esto último, resulta decisivo para la definición de nuestro foco analítico el modo en que la dimensión interaccional entre el funcionario de salud chileno y la usuaria extranjera cobra para las usuarias peruanas una importancia central en su comprensión de aquello que constituye una atención de salud de calidad. Así, desde el punto de vista de las mujeres peruanas entrevistadas, es una dimensión subjetiva la que 


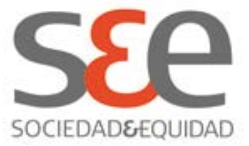

adquiere preminencia al momento de evaluar la calidad del sistema de atención materno-infantil chileno, apuntando en su condición de usuarias extranjeras a la necesidad de un trato igualitario. Factores estructurales característicos de estos contextos públicos de atención, como la sobredemanda de atención o los consecuentes tiempos de espera excesivos, son por ellas desestimados en su importancia debido a que no constituyen problemas que las afecten específicamente sino que se reconocen como problemáticas propias del funcionamiento del sistema y que afectan a todos los usuarios en general.

Así, cabe señalar que si bien las barreras que surgen en el contexto de las interacciones entre los agentes mencionados no son las únicas barreras de acceso al sistema de atención formal que enfrentan estas usuarias, otros obstáculos que influyen en la atención de las inmigrantes (como sus condiciones laborales, problemas estructurales del sistema de salud, etc.), que, sin duda tienen también una incidencia en sus posibilidades de recibir atención o cumplir con los tratamientos prescritos, serán abordados superficialmente en este trabajo. Tales limitantes emergieron con mayor recurrencia en las entrevistas dirigidas hacia los funcionarios, las mujeres peruanas, en cambio, relevaron aspectos simbólicos y no materiales de aquello que constituye la vulnerabilidad radical en que las sitúa su estatus de migrantes. También hay que especificar que consideramos como "funcionarios de salud" o "funcionarios del sistema de atención materno-infantil" tanto al personal profesional de salud como al personal administrativo.

El siguiente trabajo se divide en tres apartados; el primero describe algunos antecedentes que permiten comprender el fenómeno de la migración peruana de las últimas décadas en nuestro país. El segundo releva los hallazgos de la investigación poniendo énfasis en las narraciones de los propios actores involucrados, esto es, inmigrantes y funcionarios de salud. El tercer apartado corresponde a las conclusiones del estudio, donde se intenta dar algunas guías a partir de lo expuesto anteriormente para la interacción intercultural en el ámbito de la salud.

\section{Antecedentes de la migración en Chile}

\section{Caracterización del fenómeno migratorio}

La migración internacional en nuestro país es un proceso que si bien ha sido continuo a lo largo de nuestra historia, ha experimentado un desarrollo cualitativa y cuantitativamente significativo desde la década de los 90's (Stefoni, 2002). En el último período se ha producido la activación de un patrón de migración circunscrito a la región latinoamericana, particularmente entre los países del Cono Sur, dirigiéndose los inmigrantes a sus países fronterizos, 


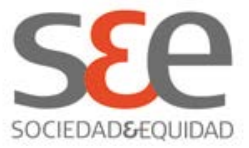

"donde el destino es una sociedad de mayor cercanía geográfica, social y cultural" (Mora, 2009: 2).

Al comparar los datos del Censo del año 2002 con los de 1992, puede notarse un incremento de inmigrantes en Chile durante la segunda mitad de la década de los noventa, época en que se desarrolla la denominada "Nueva Inmigración" (Martínez, 2003). El flujo migratorio se incrementó en un 75\% desde el año 1992 al año 2002. El 2002 existían en nuestro país 195.320 extranjeros residentes, mientras que las últimas estimaciones disponibles nos muestran que el número de extranjeros residentes en Chile es de 352 mil personas (Rivas, 2010). Esto equivale a un 2,08\% de la población nacional proyectada por el INE para el año 2009. El 61,1\% de la inmigración es de países fronterizos. Se debe destacar que en la actualidad los ciudadanos peruanos representan un 37,1\% del total de inmigrantes en Chile. Entre 2002 y 2008 ha habido un incremento de la población extranjera en un $62,3 \%$ cifra inédita para tan breve lapso. Es la población peruana la que ha mostrado el mayor aumento con un $175,2 \%$ seguida de la boliviana con un $91,1 \%$ (Agar y Saffie, 2010). ${ }^{2}$

La población peruana en nuestro país se caracteriza por ser un grupo en busca de posibilidades laborales. Según el censo del 2002, dicha población se desempeñaban principalmente en: comercio $(13,5 \%)$, servicios $(19,8 \%)$ y trabajo doméstico (43\%) (Stefoni, 2002). Cabe destacar que poco más del $60 \%$ de los inmigrantes peruanos son mujeres y el $70 \%$ de aquellas que se encontraban trabajando, lo hacían en el último sector (Stefoni, 2009). Como vemos, se ha dado una feminización de las migraciones. Si bien en el pasado las migraciones se vinculaban principalmente a hombres jóvenes, en la actualidad son cada vez más las mujeres que emigran en busca de mejores condiciones de vida, por lo general en el marco de estrategias familiares.

\section{Inmigración y Salud}

En la medida que en nuestro país se ha incrementado la relación de los migrantes con el sistema de salud público, ha surgido también el interés por investigar aquel fenómeno (Ministerio del Interior, 2008; Ministerio de Salud, 2008; Clínica Psiquiátrica U Chile, 2008; Demoscópica, 2009). Estos estudios dan cuenta de una población inmigrante que accede mayoritariamente (60\%) al sistema público de salud a través de FONASA. Las investigaciones relevan también las dimensiones a considerar en el tratamiento de los sujetos

\footnotetext{
${ }^{2}$ Acogiendo las recomendaciones de la comisión revisora del Censo 2012 (Bravo, Larragaña, Millán, Ruiz y Zamorano, 2013) hemos optado por trabajar con las proyecciones disponibles a la fecha en materia de población migrante internacional.
} 


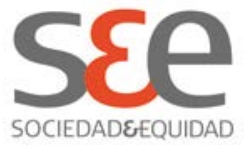

migrantes, como la situación de legalidad y/o documentación, su relación con el acceso a la atención y el financiamiento de ésta; las condiciones sociales en las que los inmigrantes se insertan; y el desconocimiento generalizado de los derechos y deberes que los migrantes tienen garantizados. Cabe destacar como los migrantes internacionales se han convertido en objeto de interés para la investigación académica e instituciones orientadas a la elaboración e implementación de políticas públicas, entre otros, que se han aproximado a ellos conceptualizándolos como suj etos de derechos.

La atención de salud pública emerge como un tópico de análisis de relevancia, por la alta recurrencia de los contactos entre migrantes y nacionales, y por la carga de subordinación que contiene la interacción entre funcionarios/as e inmigrantes (quien sabe/quien no sabe; nativo/extraño; mujer u hombre ubicada/ o en una posición de poder versus mujer en situación social y económica precaria). En éste contexto destacan los siguientes enfoques en el abordaje del acceso a la atención sanitaria por parte de la población inmigrante (Meñaca, 2004); el enfoque de los factores socioeconómicos y el de las diferencias culturales. El primer abordaje pone el acento en la situación de pobreza, marginación y exclusión en que se encuentran los inmigrantes, lo que si bien es reconocido como una variable que influye en el proceso de saludenfermedad, se relega a áreas de competencia ante las cuales los profesionales de la salud no tienen poder para actuar (lo psicológico o las condiciones de vida de las inmigrantes) (Otero, Sanz y Blasco, 2006). Bajo el abordaje de las diferencias culturales, se propone que el problema sobre el acceso no reside en la existencia de culturas distintas en los contextos sanitarios, sino en la falta de sensibilidad hacia éstas por parte de los actores involucrados en la relación. En ese sentido, Alarcón, Vidal y Neira (2003) exponen la necesidad del desarrollo de modelos de salud que respondan a las expectativas materiales y culturales del usuario, en un diálogo e intercambio cultural entre saberes, planteándose así la interculturalidad como un proceso de acercamiento entre los distintos sistemas médicos (Alarcón, Vidal y Neira, 2003).

Según Vulpiani (2000) la extensa literatura sobre la influencia de los prejuicios de los funcionarios médicos en las desigualdades de salud y de acceso a atención médica entre migrantes y autóctonos, afirma que las diferencias socioeconómicas no pueden ser la única explicación para estas realidades y subraya el hecho de cómo en algunos casos la discriminación y el racismo hacia las minorías puede ser decisivo. Pero más allá de una postura extrema como el racismo, el problema de las diferencias culturales, según Meñaca (2004) reside en gran parte en el proceso de comunicación entre funcionarios y usuarios. Al respecto el autor se refiere al concepto de competencia cultural desarrollado en Estados Unidos con el objetivo de garantizar que todo usuario del sistema de salud reciba un tratamiento efectivo 


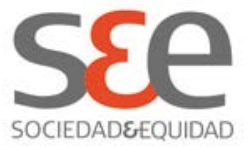

e igualitario de una manera cultural y lingüísticamente apropiada (Meñaca, 2004).

Sin embargo, se tiende a dar una sobre-sensibilización hacia el inmigrante que hace que en vez de eliminarse los estereotipos, éstos se refuercen. Según Meñaca (2004) usualmente se ha insistido en reducir la información cultural de los inmigrantes a sus culturas tradicionales, que aparecen estereotipadas y exotizadas haciéndose uso de un concepto esencialista de cultura; un conjunto de conocimientos, valores y creencias inflexibles y rígidas. De esa manera, en lugar de resultar buenas herramientas para el profesional sanitario, le pueden llevar a malentendidos y a ser más rígido que los propios migrantes en la interpretación de sus normas y tabúes culturales. Producto de lo anterior, se dibuja una situación comunicativa desigual, entre unos inmigrantes con cultura tradicional, exótica y rígida, y profesionales portadores de conocimientos no culturales, científicos y racionales (Meñaca, 2004).

Respecto a las críticas que se han hecho a ese concepto esencialista de cultura que se maneja en el sector biosanitario, Van Dijk (en Meñaca 2004) plantea que es necesario un concepto de cultura dinámico y situacional que permita incorporar los cambios culturales que el mismo proceso migratorio implica. Paralelamente, Comelles y Allué (2003) plantean la importancia de no exotizar a los pacientes inmigrantes, considerando necesario hacer ver a los profesionales del sector salud que lo cultural está presente en el contacto con todo usuario del sistema sanitario, sea o no inmigrante. Por otra parte, Meñaca (2004) plantea que también se ha propuesto a los profesionales de la medicina trabajar habilidades como la empatía, la sensibilidad cultural y la reflexión acerca de sus propios valores culturales como un ejercicio necesario para traspasar estas barreras (Meñaca, 2004).

\section{Interacción, cuerpo y racismo en el estudio de las migraciones}

El fenómeno de la inmigración va acompañado de la generación de prejuicios y estereotipos en la población local. Cuando el otro es visto como un inferior, se dificulta enormemente el proceso de aceptación, reconocimiento e integración en la sociedad receptora. La formación de representaciones culturales está asociada con la construcción del "otro/a" como alguien diferente al "nosotros/as", sin embargo, el problema aparece cuando en este proceso surgen las diferenciaciones del orden inferior/superior. Como explica Stefoni (2002), las representaciones sociales y culturales no son inocentes ni producto de las fantasías humanas, sino que responden a una lógica de poder donde los grupos dominantes refuerzan y legitiman su poder al construir a los otros como subordinados e inferiores. 


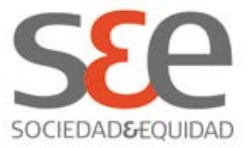

Todo lo anterior es abordable teóricamente desde las ideas de Goffman (2006) sobre el desarrollo de los "contactos mixtos", un tipo de interacciones donde existe presencia física inmediata de quienes ha denominado "normales" y "estigmatizados". Para Goffman (2006) el medio social establece normas sobre las categorías de individuos que en él se pueden encontrar; de manera que al toparnos con un extraño, las primeras apariencias nos permiten prever en qué categoría se halla y cuáles son sus atributos, a partir de lo cual nos ideamos expectativas normativas sobre él y sus conductas. Sin embargo, "el extraño" puede demostrar ser dueño de un atributo que lo vuelve diferente e incongruente con los estereotipos sobre cómo debe ser aquella determinada especie de individuos, alejándolo negativamente de las expectativas que sobre él se han puesto. Debido a lo anterior, este atributo produce un amplio descrédito de aquel extraño a los ojos de los que lo rodean; es una "indeseable diferencia", un estigma.

La manera en que se desarrolla el contacto entre "normales" y aquellos que poseen un atributo desacreditador depende en gran parte del conocimiento que tengan los normales del atributo del estigmatizado. Si la calidad de diferente del estigmatizado es ya conocida o resulta evidente para los normales, éste se encontrará en situación de desacreditado, por otra parte, si su atributo no es conocido ni inmediatamente perceptible, se encontrará en situación desacreditable (Goffman, 2006). En este sentido, toma gran importancia la reflexión sobre cuerpo y racismo desarrollada por Le Breton (1995), ya que si el atributo estigmatizador se encuentra de alguna manera inscrito en el cuerpo, en sus movimientos o maneras corporales, será fácilmente identificable y, por lo tanto, el individuo se encontrará en situación de desacreditado. Para Le Breton, el cuerpo, tal como plantea Goffman para cualquier tipo de atributos, se encuentra invisibilizado o borrado bajo una norma a partir de la cual generamos expectativas corporales de quienes nos rodean. El cuerpo se borra, se diluye en el automatismo de los rituales diarios.

Cuando el cuerpo rompe la norma, cuando se rompen las convenciones de su borramiento, la imposibilidad de los otros para identificarse con aquel cuerpo se convierte en fuente de prejuicios; la diferencia se convierte en un estigma (Le Breton, 1995). La historia individual de la persona, toda su particularidad quedan invisibilizadas bajo el cuerpo colectivo de la diferencia, bajo la raza (Le Breton, 2002). El imaginario en torno a los cuerpos es patente en los discursos médicos, y éstos son cruciales en la atención materno-infantil en salud, aún más cuando se trata de un espacio mayoritariamente femenino, lo cual conlleva otras cargas de discriminación y normalización. 


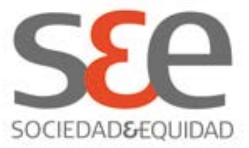

\section{Análisis}

\section{La interacción desde las mujeres inmigrantes; sus percepciones, actitudes, conductas}

Las interacciones entre inmigrantes peruanas y funcionarios del sistema de salud, que se dan durante el proceso de atención materno-infantil pueden entenderse, bajo la conceptualización propuesta por Goffman (2006) como situaciones sociales mixtas, es decir, encuentros entre estigmatizados y estigmatizadores, puesto que, siendo de por sí asimétrica la relación entre usuarios y funcionarios, a esta distinción se superpone una segunda; la de chilenos/ peruanos. En esa relación, los nacionales, representantes del grupo cultural mayoritario y dominante, son quienes además se posicionan en un estatus superior fundado en una autoridad asociada a su trabajo; mientras que las peruanas en su condición de usuarias, aparecerían como grupo estigmatizado. En este sub-apartado y en el siguiente, revisaremos los aspectos interaccionales puestos en juego en esa relación desigual que se establece entre ambos grupos de agentes que se relacionan cotidianamente en el contexto de la atención materno-infantil, abordando por separado las percepciones y conductas, tanto de las inmigrantes peruanas como de las y los funcionarios.

La dimensión del trato establecido entre usuarios y funcionarios del sistema de salud es un aspecto siempre relevante, sin embargo, parece cobrar aún más significado para un sujeto que se encuentra en una situación de vulnerabilidad como la que caracteriza a la condición migrante. Por un lado, la salud constituye un valor esencial para los inmigrantes que tomaron la decisión de asentarse en otro país por motivos económicos -que es el caso de nuestras entrevistadas- principalmente debido a que su conservación es la condición básica para poder trabajar (Fuertes Goñi y Martin Laso, 2006). A su vez, al tratarse de población económicamente activa, que se sitúa en un tramo etario de entre 15 y 50 años, también se trataría de sujetos en edad reproductiva, que demandan atención materno-infantil así como atención en salud sexual y reproductiva con miras a la planificación familiar que, como pudimos constatar a través de las entrevistas, es también un motivo de consulta recurrente entre las mujeres migrantes.

Retomando la dimensión del trato y su importancia en la evaluación de un servicio de salud, Williams, Schutt-Ainé y Cuca (2001) sostienen que éste es particularmente crucial en lo que respecta a las áreas de salud sexual y reproductiva, donde la confianza en el médico, y, en este caso, en cualquier funcionario involucrado en el proceso de atención materno-infantil, se traduciría en gran medida en apego y compromiso con las indicaciones impartidas. En ese sentido, al momento de evaluar la "calidad" de la atención 


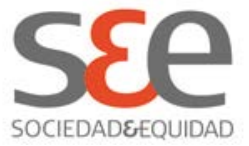

de salud, las mujeres entrevistadas se refirieron espontáneamente a una dimensión subjetiva de aquello que a su juicio constituiría una "buena atención", relevando el trato recibido como un elemento crucial de ésta. Por supuesto, también recurrieron a indicadores "objetivos", aspectos que en su mayoría fueron valorados positivamente. Estos aspectos fueron evaluados exclusivamente desde un enfoque racional y económico, es decir, haciendo énfasis en la eficacia de los tratamientos impartidos y en los beneficios prestados, los que, según las entrevistadas, eran mayores si se los comparaba con los que ofrece el sistema de salud peruano. Sin embargo, estas dimensiones que fueron evaluadas positivamente por las usuarias peruanas quedaban eclipsadas por su percepción de un trato discriminatorio recibido en el proceso de atención, el que atribuyen directamente a su condición de extranjeras. Cabe puntualizar que se advirtió en las entrevistas que las inmigrantes atribuyen el trato discriminatorio recibido a su condición de extranjeras y no particularmente a su condición de inmigrantes peruanas, pues conciben que los extranjeros en general son discriminados, o por lo menos, un amplio conjunto de extranjeros de múltiples nacionalidades.

Según Alcalde Campos (2011) el análisis de los procesos de inserción de los inmigrantes en las sociedades de llegada y los mecanismos que dan lugar a la construcción social de la figura del forastero o del extraño en esas sociedades receptoras, ha sido una problemática extensamente estudiada en el campo de la sociología de las migraciones, remontándose a las conceptualizaciones clásicas (Simmel G., (1927); Park R.E. (1928); y Schütz (1944) en Alcalde Campos, 2011). Resulta interesante cómo la definición del inmigrante, en tanto figura de alteridad, aparece estrechamente ligada a la estigmatización, entendida ésta como el despliegue de ciertas sociodinámicas que obedecen a condiciones caracterizadas por un desequilibrio de poder, procesos de exclusión y desigualdad social (Alcalde Campos, 2011). Tal como postula Goffman (2006), el estigma es un atributo que se aleja del deber ser determinado por las normas que rigen un contexto específico, por eso es una "indeseable diferencia". La visibilización de las dinámicas de conflicto social que entraña el contacto sociocultural entre extranjeros y autóctonos, da cuenta de cómo la inmigración introduce la problemática de la articulación entre la diferencia y la desigualdad (Giménez 2007: 25), pues es claro que el encuentro entre el grupo foráneo y el autóctono ocurre en un contexto que no es igualitario, desde que los grupos foráneos se sitúan en una relación asimétrica respecto de la cultura dominante.

Si el sistema de salud es para los usuarios en general, incluso nacionales, un espacio social con códigos culturales específicos que plantean una forma de hacer las cosas en la que los usuarios han de ser socializados, las diferencias culturales actuarían en el caso de las usuarias migrantes como un segundo 


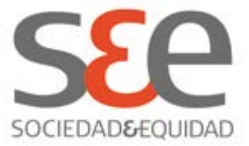

umbral de alienación (Triviño Caballero, 2012). Se observó en el discurso de las usuarias que decían sentirse desinformadas y que esa sensación se traducía la mayoría de las veces en incertidumbre y miedo. Lo más crítico de este problema es que para superar esta situación las mujeres debían recurrir, en mayor medida, a fuentes de información informales. Es decir, la iniciativa de revertir la "inadecuación" de las usuarias, evidentemente menos socializadas respecto de los mecanismos de funcionamiento de los centros de salud, no era asumida a través de medios formales que debieran hacerse cargo de las diferencias socioculturales que originan estas dificultades.

Según Liberona (2012:32) la alteridad relativa de los inmigrantes sudamericanos se convierte en alteridad absoluta cuando la interacción con los nacionales se produce en un espacio común que les exige compartir bienes y/o servicios públicos, como ocurre en el ámbito de la salud. Es en esos contextos donde el extranjero, visto como amenaza para los nacionales, aparece como un otro conflictivo. Lo anterior coincide con lo planteado por Goffman (2006), sobre cómo los estigmatizados pueden ser catalogados, en algunos casos, como perversos y peligrosos. En este caso de estudio, las mujeres peruanas manifestaron recurrentemente que los chilenos, tanto funcionarios como otros usuarios, cuestionaban la legitimidad de su demanda de atención en salud, haciéndoles sentir que abusan de un beneficio que, en su condición de extranjeras, no les correspondería recibir.

No todos, pero hay gente que trata mal, incluso en la sala de espera... los demás pacientes miran feo, como diciendo 'tú no tienes derecho a estar aquí'. (Sonia- Consultorio B).

...hay trabaj ito acá, que allá [en Perú] no hay. Pero mucha gente te mira como si le hubieras robado, salud, trabajo, todo. Es un costo alto que hay que pagar. (Beatriz-Consultorio $B$ ).

Sin embargo, a diferencia de quienes decían sentirse amedrentadas por estas percepciones, en palabras de Goffman (2006), aquellas que demuestran una internalización del estigma, hubo quienes, aunque no menos incómodas con la situación, se mostraban conscientes de que ese trato diferenciado que les impone trabas en la posibilidad de atenderse, se basa en un criterio de distinción ilegítimo, o sea, entendían esas actitudes y comportamientos como una forma de discriminación. Goffman (2006) argumenta que, a pesar de que el estigma se internalice, prevalece en el individuo estigmatizado la creencia de ser un ser humano como cualquiera, que merece oportunidades, justicia e igualdad. 


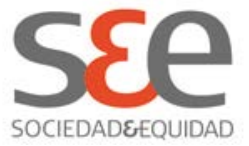

Como que a nosotros los inmigrantes no somos como valorados y... como no se po', todos somos iguales digo yo... (Paulina - Consultorio B).

Sin duda, desde el punto de vista de las usuarias peruanas, la categoría de "inmigrante", "extranjero" y todas sus variantes, asume en este contexto una muy potente carga valorativa. Desde el punto de vista de Le Breton (1995), cuando se habla de inmigrante se atribuye con esa palabra una especie de condición, de esencia, más que una situación legal; se trata de un atributo puesto desde fuera, un estigma que se sustenta en una ideología de deshumanización de ese otro inmigrante, de ahí que las peruanas, al reconocer esa infravaloración, expresen su necesidad y derecho de ser vistas y tratadas como iguales a los chilenos.

He escuchado, 'pero son de otro país, cuando se van a dar a luz, cuando van a dar el parto'... no tienen buena atención, las gritan... 'para qué vienen a dar a luz acá, que se embarazan, que esto, que lo otro', como que asustan un poco... pero yo les digo que tampoco hay que dejarlos, si hay que hacer valer nuestros derechos si todos somos iguales, a final de cuentas uno paga imposiciones, paga todo acá... (Laura- Consultorio A).

Como señala Goffman (2006) el portador del estigma puede no sentirse compungido o impresionado por ello. Sin embargo, igualmente enfrentará las consecuencias materiales de la dominación, exclusión y desigualdad. En ese sentido, gran parte de las mujeres entrevistadas afirmaron que en muchos casos son los funcionarios, principalmente el personal administrativo, quienes les ponen obstáculos en el acceso a atención, aun cuando esa disposición contraría la política de atención universal a la que, en teoría, adscriben los centros de salud en materia de atención materno-infantil.

La primera vez cuando vine aquí con la guagua abajo [donde se inscriben las usuarias], me dijeron que no, que no... Acá no te atienden, la matrona sí, pero allá abajo no... Yo tengo entendido que aquí a los inmigrantes no los atienden por más que ponen ahí, coloquen avisos, diferentes carteles, pero yo tengo entendido que no los atienden y ponen dificultades, si a mí no más me atienden por la guagua... (ÁngelaConsultorio B). 


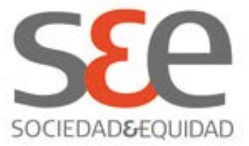

- ¿Y usted sabía que a cualquier mujer embarazada, no importa si está legal o no, se la tiene que atender?

- No, yo no sabía... mire, cuando yo vine reciencito pa' acá, pa'l consultorio, me dijeron, porque yo quería cuidarme, no quería tener bebé, entonces por la situación, que no tenía para comprarme los anticonceptivos, ¿no?...entré acá y me dijeron que no, querían el carnet, el pasaporte... (Beatriz- Consultorio B).

Este problema en torno al acceso nos remite a la temática de la consideración de los migrantes como sujetos de derecho. En el ámbito de la salud, nos encontramos con que si bien a nivel internacional, legalmente se considera la salud como un derecho inalienable para toda persona, sin importar su situación migratoria, en la práctica este derecho es sucesivamente vulnerado (Liberona 2012: 22). Como señalan Núñez y Torres (2007) en el caso chileno, el acceso a la salud de la población migrante se supedita al tipo de visa que se tenga. Aquellos que tienen un permiso de trabajo o cuentan con residencia permanente tienen la posibilidad de cotizar en el sistema público o privado, en cambio, quienes no han regularizado su situación migratoria no tienen derecho de atención en los servicios de salud pública, a menos que se trate de una urgencia, atención de parto, mujeres embarazadas o niños menores de seis años.

En el curso de esta investigación observamos que el frecuente incumplimiento de esas disposiciones actuó, tal como señalaron algunas mujeres, como un factor que desincentivó su oportuna concurrencia a los controles del embarazo, pues aseguraban tener conocidas a las que previamente se les negó la atención, por lo que temían correr esa misma suerte:

Bueno, la primera vez yo decía cómo me van a atender cuándo quedé embarazada. Pasé hasta los dos meses sin consultar, casi hasta los tres meses sin consultar. Después me dijeron tienes que irte a controlar, porque el bebé puede estar mal y vine... fui a migraciones y ahí ya le conté mi caso... 'oye, señorita, no tengo trabajo, estoy embarazada', pues porque me habían dicho 'no te van a atender, te van a cobrar en el hospital, no te van a atender así por así, porque tienes que tener tus papeles'. Fui al consultorio y me dijeron, 'sí, te van a atender, tramita tus papeles'... y yo dije, 'pero no tengo quien me haga la contrata y 


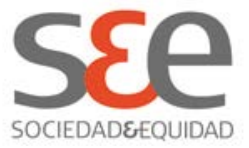

necesito sacar mis papeles'... 'ya', me dijeron, me dieron el 'manda' ${ }^{3}$ que me dieron, que me dijeron que mandara por correo para recibir la temporaria. (Emilia -Consultorio A).

En lo que se refiere al trato con los funcionarios de los centros de salud, como ya se ha dicho, ellas perciben un tratamiento discriminatorio que atribuyen a su nacionalidad extranjera. Debido a esto hay quienes se sienten marginadas e invisibilizadas, teniendo esta situación una incidencia negativa, por ejemplo, en la oportunidad de acceder a la información acerca de prestaciones y beneficios a los que pudieran optar.

En el trato se hacen diferencias, sobretodo porque no te responden, hacen como que no te oyeran, como que no existes, no contestan, no dan información... aquí todo hay que preguntar porque no te dicen nada...hay mucha marginación acá para uno que es extranjera, es triste... (Beatriz- Consultorio B).

En oposición a esa invisibilización acusan en otro sentido la existencia de una suerte de hipervisibilidad. Las mujeres peruanas, al sentirse fácilmente identificables como extranjeras, de algún modo se sienten expuestas. En su caso, lo evidente de los atributos que las diferencian o la visibilidad del estigma, como diría Goffman (2006), las pone inmediatamente en la situación de sujetos desacreditados desde el punto de vista de los "normales", en este caso, funcionarios y usuarios chilenos.

Un poco te miran mal, porque saben que tú eres extrajera y te miran, 0 te quedan mirando... (Beatriz- Consultorio $B$ ).

El sentirse permanentemente observada, la sensación de estar "en exhibición" que puede tener lugar en una situación de contacto mixto, pone de manifiesto cómo el individuo estigmatizado se mantiene alerta a lo que los demás consideran su "defecto", percibiendo ese atributo como una posesión impura que posiblemente le avergüenza, pues al haber incorporado las pautas de la sociedad más amplia, acepta que está lejos de "ser como debería" (Goffman 2006). Respecto a esa visibilidad de la diferencia, Le Breton (2002) afirma que la discriminación, al vincularse a rasgos fácilmente identificables impone una versión reificada del cuerpo. "La presencia del Otro se subsume bajo la de su cuerpo. Él es su cuerpo. La anatomía es su destino" (Le Breton

\footnotetext{
${ }^{3}$ Oficio, documento.
} 


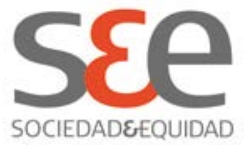

2002: 76). Así, la historia individual de la persona, toda su particularidad, quedan invisibilizadas bajo el cuerpo colectivo de la diferencia, bajo la raza.

En las entrevistas, la dimensión de la apariencia, entendida como "medio deliberado de difundir una información sobre uno mismo" (Le Breton 2002: 82), de presentarse frente al otro, cobra importancia para las inmigrantes como un elemento decisivo en el trato, ya no sólo referida a un origen racial/nacional sino también a otras dimensiones implicadas en la manera de presentarse en la interacción, surgiendo aspectos como la vestimenta.

- ¿Cómo siente usted que tratan en general los médicos a las personas, independientemente si son inmigrantes? ¿O hay algunas diferencias que puedan establecer?

- Depende ¿no?, con todos, son algunos que de repente algunos te miran por la... no sé, cómo te ven, por la vestimenta, o no sé yo, si ven a alguien mejor vestido creo que es mejor trato, pero si te ven más o menos como que... 'ya, pasa por aquí' [de mala manera]... pero no puedo catalogar a todos también por iguales, no son todos, no, rara vez, pero... a veces no tienen paciencia... te atienden, a veces tú no sabes las cosas de repente de lo que te explican... como que te tratan mal... Y no solamente acá, allá en Perú es igual también... como te ven vestido así te atienden... (Laura - Consultorio A).

La presentación física parece valer como una presentación moral del individuo (Le Breton, 2002), que justifica un actuar diferenciado por parte de quién evalúa dicha apariencia. En este caso, el prejuicio deviene en un mal trato $y$, según el entrevistado, éste se focaliza en el indicador vestimenta, lo que podría hablar de un prejuicio de clase, situación que se daría tanto en Chile como en Perú.

\section{El funcionario al interior de la interacción; sus percepciones y conductas}

Como hemos planteado anteriormente, las usuarias inmigrantes afirman enfrentarse a frecuentes tratos discriminatorios en el proceso de atención materno-infantil, maltratos cuya existencia es también reconocida por los funcionarios. A pesar de lo anterior, los funcionarios nunca admiten las acciones discriminatorias en la forma de una autocrítica, sino que siempre las adjudican a otros funcionarios. El tratamiento distante de tales situaciones ofrecido por los funcionarios, que sólo se limitan a la constatación de su 


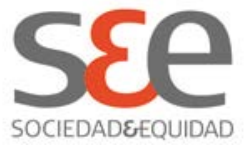

ocurrencia, contrasta con los relatos emocionalmente cargados de las inmigrantes.

Ya se ha afirmado que las mujeres peruanas al sentirse fácilmente identificadas como extranjeras, se sienten expuestas. En la interacción con los funcionarios, las usuarias inmigrantes demuestran o visibilizan su nacionalidad u origen peruano; atributo que las desacredita ampliamente a los ojos de éstos. Al conocer su atributo, los estigmatizadores dejan de ver al inmigrante como "una persona total y corriente para reducirlo a un ser inficionado y menospreciado" (Goffman 2006: 12). La "peruanidad" es, por lo tanto, un estigma. Al poseer las usuarias este estigma, dejan de ser totalmente humanas (Goffman, 2006) y esta deshumanización justifica, desde la perspectiva de los funcionarios, los tratos discriminatorios que hemos constatado que se les dan. La estigmatización de los migrantes supone, como diría Le Breton (2002: 76), "un ejercicio perezoso de clasificación"; cada inmigrante es un eco incansablemente repetido del gigantesco "cuerpo inmigrante" y a favor de ese cuerpo colectivo queda invisibilizada su historia individual, su diferencia, su particularidad (Le Breton, 2002). La nacionalidad resulta simbolizar todo un conjunto de caracteres; sostiene una representación acerca de cómo son quienes comparten ese origen.

Desde la perspectiva de Goffman (2006), un atributo indeseable se transforma en estigma cuando es incongruente con nuestro concepto sobre cómo debe ser determinada especie de individuos. En este sentido, el 'deber ser', la norma, es un elemento fundamental de la perspectiva del estigmatizador. En efecto, cuando no podemos identificarnos con el otro, éste "deja de ser un espejo tranquilizador de la identidad, abre una brecha en la seguridad ontológica que garantiza el orden simbólico" (Le Breton, 1995 :136). El inmigrante como figura de alteridad pareciera cuestionar los ideales normativos naturalizados. En este apartado interesa adentrarnos en las percepciones de los funcionarios sobre las usuarias inmigrantes peruanas, en aquella representación de las inmigrantes que se construye tras el significante "peruana", ya que, si bien tienden a atribuírsele sin razón al estigmatizado un elevado número de imperfecciones, interpretándose cualquier actitud de ellos de forma negativa y como una expresión directa de su calidad de diferente (Goffman 2006), algunas de las percepciones sobre el estigmatizado que presentaremos a continuación parecieran reflejar ciertos ideales normativos puestos en cuestionamiento.

A ojos de los funcionarios, algunas de las características de las inmigrantes peruanas son su "lentitud" y su menor capacidad para "captar" o "retener" la información. 


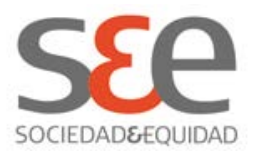

... en el tema de la dación de horas e inscripciones ahí se pierde un poco la información, aunque uno les diga cómo que no, no sé si no la captan... o... (Gabriela, funcionaria administrativa).

...Porque, porque son... son... como dije denante, influye mucho la parte educacional, entonces y entienden... mal, ¿me entiendes? No, no, cuándo tú les das la información, ellos como que no retienen la información, entonces, "ah, señorita", "¿pero no vino antes ayer al médico?", "sí señorita, pero es que no le dije a la doctora que me dolía el dedo", es un ejemplo... "pero cómo si yo le dí hora el lunes", "sí, pero es que se me olvidó decirle a la señorita que me dolía el pie y le dije solamente que me dolía la cabeza y lo que más me molesta es el pie", ¿me entiendes?, entonces se van repitiendo, se van repitiendo... (Adela, funcionaria administrativa).

Ya se ha sostenido que las migrantes pueden encontrarse menos socializadas en el funcionamiento de los centros de salud, y que las diferencias socioculturales y la vulnerabilidad socioeconómica -entre otros factores-, se superponen, influyendo en la adecuación de estas usuarias al sistema. Sin embargo, estas consideraciones no se hallan en el discurso de los funcionarios, quienes se refieren, en cambio, al "bajo nivel educacional" de las migrantes. Estas alusiones resultan ser parte de aquella ideología construida por los estigmatizadores para explicar la inferioridad y deshumanización de los estigmatizados; lo que Goffman (2006) Ilama "teoría del estigma". A pesar de que esta teoría justifica su deshumanización, pudieron notarse durante las entrevistas los esfuerzos de los funcionarios por formular su opinión de una manera políticamente correcta, elaborando eufemismos como lo es la expresión "no retienen la información". Aun así, planteaban su punto de vista de una manera burlesca, tal como queda plasmado en la parodia realizada por la funcionaria para narrar la 'excesiva' asistencia de las inmigrantes al servicio de salud.

La molestia patente por la policonsulta sería una expresión del disgusto que produce en los funcionarios el hecho de que las inmigrantes ejerzan sus derechos en salud. De aquí se releva que existe la percepción de que a ellas no les corresponde utilizar esos servicios y, por lo tanto, que la policonsulta constituye un abuso. 


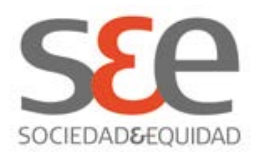

Hay discriminación y eso no se puede negar, hay discriminación por parte de los funcionarios, más que todo porque llegan muchos inmigrantes con un nivel educacional alto, entonces ellos saben a lo que tienen derechos, y como que a ellos les molesta un poco que ellos sepan, o sea, exigen, pero en realidad es parte, digamos, de su derecho de salud... (Gabriela, funcionaria administrativa).

Otras percepciones sobre los inmigrantes peruanos son que, por un lado, son "groseros", "atrevidos", "prepotentes" y "agresivos", y, por otro lado, les cuesta adaptarse a las normas del sistema de salud. Muchas de estas apreciaciones se realizan cuando los funcionarios se refieren a la exigencia de los derechos de salud por parte de los inmigrantes, aludiendo a que los "exigen groseramente" y de "mala forma" o que demandan sin considerar las normas internas.

- Lo que pasa es que ya cuando ya están acá dentro del sistema, exigen más que cualquier otra persona, más que los chilenos (...)

- Pero las cosas que exigen, en el fondo, ¿están bien?

- Sí, sí, pero las exigen de repente, groseramente o de mala forma, a eso me refiero...

(Adela, funcionaria administrativa).

...Vienen muchos, son bastante demandantes y les cuesta adaptarse a las normativas del consultorio. Llegan tarde, en las consultas si pasan 20 minutos y la persona no está no se le atiende y ellos exigen la atención. No respetan mucho las normas internas. Las normas se les explican bien, el problema es que faltan mucho. Permanentemente van yendo a su país, faltan a los controles y no avisan que no van a estar con tiempo... (Daniela, funcionaria administrativa).

Todas estas apreciaciones negativas sobre la inmigrante peruana (su "lentitud", su "prepotencia", su "agresividad", su abuso del sistema), parecen revelar una concepción sobre cómo debe ser el usuario ideal de los consultorios; un usuario chileno cuya nacionalidad lo hace merecedor de los beneficios del sistema de salud, un usuario que no exija mayor esfuerzo de atención por parte del funcionario, un usuario que no "de problemas". Un 


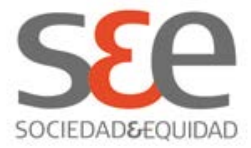

usuario que esté familiarizado con el lenguaje, con los modismos, con el funcionamiento del sistema de salud, etc., y que, por lo tanto, no necesite de mayores instrucciones.

Cuando la interacción de los inmigrantes con los nacionales se produce en un espacio común que les exige compartir bienes y/ o servicios públicos, el extranjero, visto como amenaza para los nacionales, aparece como un otro conflictivo (Liberona, 2012). A esto hay que sumar que la molestia frente a la exigencia por parte de las inmigrantes de sus derechos en salud, la sensación de que abusan de algo que no les corresponde, cobra especial relevancia en el contexto de la atención materno infantil. La mujer inmigrante y procreadora se enmarca en discursos sobre embarazadas eternas y cargadas de criaturas como imágenes del terror que alientan las políticas regulatorias de población. Estos discursos representan a las mujeres inmigrantes conminando el bienestar, los indicadores de salud, los sueldos de los funcionarios; amenazando con el aumento de la población peruana en el país, la que cada vez se apropia de más beneficios, tanto de salud, como de educación o trabajo, beneficios que no les corresponden y que debiesen repartirse sólo entre los nacionales. A partir de estos discursos se genera la idea de que son usurpadores; abusando del sistema de salud, quitándole el trabajo a los chilenos, etc. De esta manera se dibujan concepciones cada vez más negativas del inmigrante peruano y en este punto toma sentido recoger lo que plantea Goffman (2006) al afirmar que los estigmatizados, en casos extremos, pueden ser catalogados como perversos y peligrosos, lo que genera reacciones de parte de los estigmatizadores que son más discriminantes y violentas. Reacciones como aquella relatada por una inmigrante, que afirmó que un funcionario se dirigió a ella afirmando que las peruanas eran "fáciles" para tener hijos.

En aquel perezoso ejercicio de clasificación que constituye la estigmatización se acoplan lo ético y estético, transformándose el cuerpo en un signo fatal de defectos morales (Le Breton, 2002). Así, el embarazo, en el contexto de aquel cuerpo éticamente defectuoso, pasa a concebirse como igualmente perverso, como una consecuencia del ser una "mujer fácil". Sumándose a lo anterior la representación de que las peruanas tienen hijos por conveniencia, buscando los diversos beneficios que aquello pueda suministrar, como la agilización de los trámites de regularización. Se observa entonces la superposición del estigma de la peruanidad con la violencia de género (Segato, 2003).

Como se ha planteado con anterioridad el individuo estigmatizado rompe con ciertos ideales normativos del estigmatizador. Foucault (2002) plantea que la disciplina, como forma de poder y control, normaliza los cuerpos; busca que se ajusten a una norma de cómo se debe ser, hacer, 


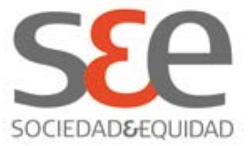

producir. En este sentido, como lo ha planteado Butler (2002), el sexo y la raza se transforman en categorías normativas, Io que Foucault (1977) Ilamó “ideal regulatorio". Lo que significa que sexo y la raza funcionan como norma y como práctica regulatoria, cuya fuerza se manifiesta en el poder de producir demarcar, circunscribir, diferenciar- los cuerpos que controlan. Una norma cultural donde actúan los prejuicios y operan los discursos en torno al cuerpo: olores, colores, texturas, prácticas de higiene, prácticas sexuales, etc. (Butler, 2002). En relación con esto, otra apreciación sobre las inmigrantes peruanas es que son "sucias" y no cuidan su higiene ni la de sus hijos. Nuevamente la estigmatización apunta hacia un deber ser naturalizado; Ias normas sanitarias.

Nosotras hacemos control sano, entonces vemos niños, les tenemos que revisar los pies, el pelo, los dientes... entonces se ve que a veces tienen poco hábito de limpieza... (Karen, funcionaria profesional de la salud).

Cabe mencionar que el discurso de los funcionarios suele oscilar entre dos posturas contradictorias. Alternan entre actitudes de crítica y molestia hacia los inmigrantes peruanos, momento en el cual dejan fluir todas sus apreciaciones negativas y sentimientos de disgusto; y posturas comprensivas, momento en el cual justifican de diversas maneras la forma de ser que le atribuyen a los migrantes y formulan ciertas percepciones positivas acerca de ellos. Se advierte la necesidad de los entrevistados de matizar su discurso para instalarse en una posición que tienda a lo "políticamente correcto". En relación con lo anterior, un aspecto mencionado frecuentemente por los funcionarios y que se podría considerar como parte de la "teoría del estigma", fue el de las condiciones de vivienda de los inmigrantes peruanos, aludiendo al déficit que presenta su infraestructura y la condición de hacinamiento, repercutiendo directamente en las condiciones sanitarias y la salud de las inmigrantes y sus familias.

Bueno, ahí la cosa es un poco más complicada para ellos, porque como viven en, en cuartos, en piezas, no tienen en esas piezas un lavamanos, tienen cocinas comunes, en esas casas dónde viven, cuando hacemos las visitas lo podemos ver, tienen un baño común, una cocina común, entonces difícilmente pueden tener higiene como nosotros quisiéramos (...) una llave para lavarse las manos cada vez que mudan el niño o (...) tener una llave dónde poder lavar sus mamaderas, esterilizarlas bien, etc., o sea, en eso, ellos están un poco mal, por las condiciones de la vivienda que tienen... (Patricia, funcionaria profesional de la salud). 


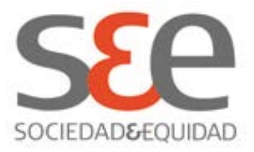

Porque no solamente tienen carencias de salud, tienen carencias económicas, carencias afectivas, carencias de convivencia... viven hacinados... que uno se asombra, porque tú no te explicas que en una pieza grande puedan vivir, que de repente tú te quedas atónita cuando te cuentan que en una pieza pueden vivir hasta dos o tres familias... Entonces tú te das cuenta que, dentro de todo, son gente que sufre, que ha venido de su otro país pa' poder mejorar su condición económica y resulta que acá igual se encuentran con una realidad que es difícil... (Adela, funcionaria administrativa).

También surgen en el discurso de los funcionarios ciertas consideraciones positivas o neutrales sobre las inmigrantes peruanas; se les considera "sumisas", "tranquilas" (en oposición a la actitud acelerada del chileno), "respetuosas", "obedientes", "tímidas", "apacibles", y que "siguen las indicaciones y normas". Estas apreciaciones parecieran ser contradictorias con las antes mencionadas, pero ya hemos planteado que el discurso de las funcionarias se caracteriza por oscilar entre lo crítico y lo políticamente correcto. Por otro lado, Goffman (2006) ha sostenido que las actitudes del estigmatizado tienden a ser paradójicas y cambiantes, alternando entre el retraimiento defensivo y la agresividad. Según este autor, sabiendo lo que puede enfrentar el estigmatizado en una situación social mixta, puede responder anticipadamente con un retraimiento defensivo, mientras que en otros momentos, en vez de retraerse, puede mostrarse anticipadamente violento. Además, el estigmatizador tiende a atribuirle al estigmatizado, además de un elevado número de imperfecciones, ciertos atributos positivos pero no muy deseados (Goffman, 2006).

...yo les tengo como cierta capacidad de admiración porque son de una tranquilidad algunos, en general, son muy pocos los que te dije en denante, que eran muy pocos algunos agresivos... pero ellos como personas son gente apacible, no como nosotros los chilenos que somos ¡pum! súper acelerados, no, ellos son gente apacible, yo los encuentro gente, gente tranquila... (Adela, funcionaria administrativa).

No, ellos son tremendamente, tremendamente respetuosos, ellos son muy respetuosos, son gente respetuosa per se o sea, no porque sean ellos, o sea son extranjeros y, a mi modo de ver, los veo gente muy 


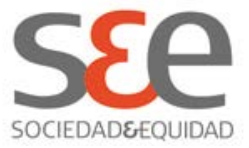

respetuosa, que acatan las normas, las indicaciones, ellos se dirigen

bien acá, a la gente... (Patricia, funcionaria profesional de la salud).

Habiendo revisado por separado las percepciones y conductas tanto de las inmigrantes peruanas como de las y los funcionarios con los que interactúan en el proceso de atención materno-infantil, se puede plantear que nos encontramos ante una relación sumamente compleja, dónde los estereotipos, prejuicios, representaciones y expectativas mutuas están intensamente presentes y toman una relevancia fundamental. Debido a toda la carga de representaciones que se apoya sobre estos contactos mixtos, suelen ser incómodos y molestos para los involucrados. Como plantea Goffman (2006:30):

Sentimos que el individuo estigmatizado percibe cada fuente potencial de malestar originada en la interacción, que sabe que también nosotros lo percibimos e incluso que sabemos que él lo sabe. Ya están dadas, pues, las condiciones para el eterno retorno de la consideración mutua.

\section{Reflexiones finales}

Nos hemos concentrado particularmente en el análisis de las actitudes y percepciones desde y hacia dos de los agentes involucrados en el proceso de atención materno-infantil: inmigrantes y funcionarios de la salud. En los primeros, identificamos como factores obstaculizadores hacia el acceso a la atención: el (des)conocimiento de los mecanismos de funcionamiento e información de las instituciones; que el trato, en sus dimensiones subjetiva y racional-económica, incide en las diferencias en la integración; el cuerpo de los sujetos en tanto se asocia a la (in/ hiper) visibilización del colectivo peruano y su pertenencia a estratos socioeconómicos bajos. En el discurso de los segundos, identificamos la operación de una situación social mixta (Goffman, 2006). En el discurso de los sujetos ${ }^{4}$ aparece la relación entre estigmatizadores (funcionarios y usuarios chilenos) y estigmatizados (inmigrantes peruanos) guiados por la actualización de representaciones que obedecen a la lejanía/cercanía de los ideales normativos, alimentados además por la construcción social de la "peruanidad", con las particularidades que ella adquiere en la sociedad chilena.

\footnotetext{
${ }^{4}$ También se incluyeron en el análisis ciertas alusiones a interacciones entre usuarios chilenos y usuarias peruanas que surgieron como relevantes, interacciones que no habían sido consideradas en el estudio en un primer momento y que también se definen como contactos mixtos.
} 


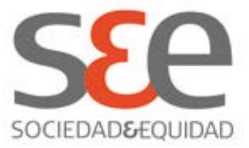

Se ha planteado que nos encontramos ante una interacción sumamente compleja, dónde los estereotipos, prejuicios, representaciones y expectativas mutuas están intensamente presentes y toman una relevancia fundamental. De esta manera es posible proponer un modelo relacional en el que no hay "victimarios" ni "victimas", sino la permanente construcción y actualización de esquemas de interacción y de representaciones basados en una relación de estigmatización. En este sentido se sostiene que el funcionario es también objeto de prejuicios y representaciones negativas a partir de las cuales se generan expectativas y respuestas.

Rescatamos el carácter emergente de las barreras, en tanto producto social resultante de las relaciones establecidas entre los actores en este particular contexto de atención, que se nutre y articula con otros contenidos sociales, tales como la construcción social del "otro peruano" existente en la sociedad chilena, y la construcción social del usuario en un contexto de atención biomédico.

Cobra relevancia el hecho de que en estas usuarias no hemos constatado, a diferencia de lo esperado, significativas diferencias culturales, en lo referente a concepciones y prácticas sobre sistemas y modelos médicos. Las indicaciones y prácticas médicas recomendadas en el SSMC, no parecían, al menos en el ámbito discursivo ser concebidas bajo la lógica de la contradicción o complementariedad respecto de prácticas particulares que las inmigrantes llevaran a cabo estando embarazadas o en el cuidado de los primeros meses de vida de los hijos. Por otro lado, resulta interesante que la concepción que tienen las usuarias peruanas de una buena atención en la dimensión del trato, lejos de aludir a una necesidad de un trato pertinente a particularidades culturales que no reconocen tener, apunta en realidad a demandar un trato igualitario, el que en su condición migrante se juega en el plano de su reconocimiento como ciudadanos. En ese sentido, afirmamos la necesidad de redefinir las actuales orientaciones hacia un modelo de atención que considere las particularidades del sujeto migrado en un modelo que proponemos como con pertinencia a la condición migrante.

El concepto de condición migrante que hemos elaborado contempla básicamente una dimensión material y una simbólica, constituyéndose ambas como obstáculos para la oportunidad de las usuarias migrantes de tener acceso a una atención de calidad en salud. La dimensión material comprende las condiciones de vida de estas mujeres y sus familias, particularmente sus precarias condiciones de habitación marcadas por el hacinamiento así como también los contextos socio-laborales en los que se desenvuelven, donde se evidencia recurrentemente la vulneración de sus derechos. En cambio, la dimensión simbólica, que es la que representó el foco de esta investigación, 


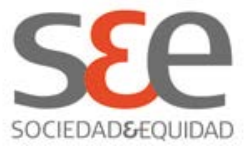

hace referencia al plano de las representaciones socioculturales, el modo en que operan las dinámicas de estigmatización sostenidas, en este caso, en una construcción social del extranjero como categoría de alteridad. La suma de los anteriores elementos incide en el autoposicionamiento que los migrantes realizan para relacionarse con la institucionalidad y el conjunto de suj etos que están presentes en las diversas interacciones potencial y actualmente realizadas en el proceso de atención materno-infantil.

Un modelo con pertinencia a la condición migrante debiera problematizar el modo de otorgar cobertura y atención efectiva, involucrando entonces aquellos elementos que dificultan el acceso e inciden en la interacción, pues aun cuando no surjan directamente de ella la afectan.

Entre aquellos aspectos susceptibles de intervención directa por parte de los funcionarios de salud se encuentra el trato y acceso igualitarios en el sentido de una manera de relacionarse basada en el ejercicio de derechos humanos y la no discriminación, respecto de las usuarias nacionales.

La otredad se inscribe en relaciones de poder que producen desigualdad, por ello resulta necesario incorporar en el modo de prestar las atenciones una concepción centrada en el paciente como un individuo, no como ejemplo de un estereotipo, como un ser humano que se enfrenta al peligro y la incertidumbre, no sólo un caso, sino como una oportunidad del médico para participar en un elemento esencial de la tarea bioética de proporcionar bienestar.

Estos elementos de carácter simbólico-relacionales son de gran relevancia en el momento en que las usuarias efectúan evaluaciones sobre la calidad de elementos materiales, $y$, consideramos, pueden ejercer implicancias en el cumplimiento de las metas hacia las cuales se orienta el SSMC u otras instituciones de atención con problemáticas similares en tanto receptoras de usuarios migrantes.

\section{Referencias Bibliográficas}

Agar, L. y Saffie, N. (2010). Migrantes en Chile: políticas públicas en salud, cohesión social e interculturalidad. En L. Agar (Coord.), Migraciones, salud y globalización: entrelazando miradas (pp. 51-64). Santiago, Chile: Biplano.

Alarcón, A., Vidal, A. \& Neira Rozas, J. (2003). Salud intercultural: elementos para la construcción de sus bases conceptuales. Revista Médica Chile, 131 (9): 1061-1065. doi: 10.4067/ S0034-98872003000900014. 
Alcalde Campos, R. (2011). De los outsiders de Norbert Elias y de otros extraños en el campo de la sociología de las migraciones. Papers. Revista de Sociología. 96 (2), p. 375-387. Disponible en http:// papers.uab.cat/article/ view/v96-n2alcalde/ 378

Butler, J. (2002). Cuerpos que importan: sobre los límites materiales y discursivos del sexo. Buenos Aires, Argentina: Paidós

Bravo, D., Larragaña, O., Millán I., Ruiz, M. y Zamorano, F. (2013) Informe Final Comsión Externa Revisora del Censo 2012. Recuperado de http:// www. censo.cl/ informe final.pdf

Comelles, J., Mascarella. M.y Allué, X. (2003). Estado del bienestar y culturas organizacionales. Efectos de las migraciones extracomunitarias en el sistema de salud español. Ponencia presentada en Medical Anthropology at Home. Medical anthropology, welfare state and political engagement. Perugia, Italia.

Comisión Económica para América Latina y el Caribe (CEPAL) (2007). Migración internacional, derechos humanos y desarrollo en América Latina y Caribe. Disponible en División de Población CELADE: http:/ / www.eclac.cl/ publicaciones/ xml/ 8/ 26608/ LCW98-migracion.pdf

Demoscópica. (2009). Diagnóstico y factibilidad global para la implementación de políticas locales de salud para inmigrantes en la zona Norte de la región metropolitana. Disponible en Ministerio de Salud

http:// www. redsalud.gov.cl/ portal/ url/ item/ 71841d2e91f518ale04001011f015 cc6.pdf

Femenías, M. y Soza Rossi, P. (2009). Poder y violencia sobre el cuerpo de las mujeres. Sociologías, 11 (21), 42-65. Disponible en http:// www. scielo.br/ pdf/ soc/ n21/ 04.pdf

Fernández Tapia, C. (2009). Síntesis estudio de caracterización de comunidades peruanas de la zona norte de Santiago. Cultura Urbana 6 (25) 1-17. Recuperado de http:// cultura-urbana. cl/ sintesis-estudio-de-caracterizacion-decomunidades-peruanas-en-la-zona-norte-de-santiago/

Foucault, M. (1977). Historia de la Sexualidad. Volumen I: La Voluntad de Saber. Madrid, España: Siglo XXI.

Fuertes Goñi, C. y Martín Laso, M.A. (2006). El inmigrante en la consulta de atención primaria. An. Sist. Sanit. Navar. 29 (Supl. 1), 9-25. Disponible en

http:// scielo. isciii.es/ pdf/ asisna/ v29s1/ original1.pdf 


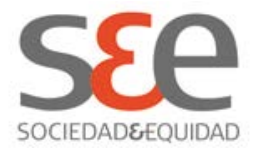

Giménez, C. (2007). Migración, sociedad y cultura: La perspectiva antropológica. En Lison Tolosana C. (Ed), Introducción a la antropología social y cultural-teoría, método y práctica. (pp. 153-190). Madrid, España: Akal.

Goffman, E. (2006). Estigma: la identidad deteriorada. Buenos Aires, Argentina: Amorrortu.

Le Breton, D. (2002). La sociología del cuerpo. Buenos Aires, Argentina: Nueva Visión.

Le Breton, D. (1995). Antropología del cuerpo y modernidad. Buenos Aires, Argentina: Nueva Visión.

Liberona Concha, N. (2012). De la alterisación a la discriminación en un sistema público de salud en crisis: conflictos interétnicos a propósito de la inmigración sudamericana en Chile. Revista de Ciencias Sociales ( $\mathrm{Cl}$ ), (28) 19-38. Disponible en http:/ / www. redalyc. org/ articulo. oa?id=70824554002

Martínez Pizarro, J. (2003). El encanto de los datos. Sociodemografía de la inmigración en Chile según el censo de 2002. Disponible en Comisión Económica para América Latina y el Caribe: http:// www.eclac.org/ publicaciones/ xml/ 2/ 14312/ Icl2046-P.pdf

Mora, C. (2009). Estratificación social y migración intrarregional: algunas caracterizaciones de la experiencia migratoria latinoamericana. Universium 24 (21), 128-143. doi: 10.4067/ S0718-23762009000100008.

Meñaca, A. (2004). Salud y Migraciones. Sobre algunos enfoques en uso y otros por utilizar. En G. Fernández J uárez (Ed), Salud e interculturalidad en América Latina. Perspectivas antropológicas (pp 53-64). Quito, Ecuador: Abya-Yala.

Ministerio del Interior (2008). Estudio de Inmigración, Equidad de género y salud pública. Principales resultados. Disponible en

http:// www.interior.gov.cl/filesapp/seminario_migraciones_13_11_08.pdf

Ministerio de Salud (2008). Atención en salud de población inmigrante en riesgo social y situación de permanencia no regular. Disponible en

http:// www.redsalud.gov.cl/ portal/ url/ item/71821af88f80fda7e04001011f0164 f3.pdf.

Núñez, N. y Torres, C. (2007). Mujeres migrantes peruanas y salud reproductiva. Usuarias de consultorios de salud de la zona norte de la Región 


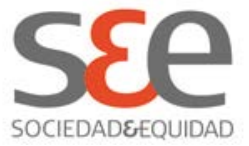

Metropolitana. Disponible en Instituto de la Mujer: http:// www.insmujer.cl/ Estudio_Mujeres_migrantes.pdf

Otero García, L., Sanz Barbero, B. y Blasco Hernández, T. (2006). La salud de la población inmigrante desde el discurso científico biomédico. Recuperado de http:// www. universidaddelasalud. es/ pdf/ investigacionysalud/ pdf/ LA\%20SALUD $\% 20 D E \% 20$ LA\%20POBLACION\%20INMIGRANTE\%20DESDE\%20EL\%20DISCURSO\%20CIE NTIFICO\%20BIOMEDICO.pdf

Rivas, H. (2010). En Migraciones, salud y globalización: entrelazando miradas. En L. Agar (Coord.), Migraciones, salud y globalización: entrelazando miradas (pp 65-86). Santiago, Chile: Biplano.

Rojas Castillo, G. (2008). Diagnóstico y factibilidad global para la implementación de políticas globales de salud mental para inmigrantes de la zona norte de la Región metropolitana. Disponible en Ministerio de Salud:

http:// www.redsalud.gov.cl/ portal/ url/ item/ 71821af88f6ffda7e04001011f0164 $\underline{\text { f3.pdf }}$

Ruiz, E. (2008). Migración y desarrollo en América latina: ¿círculo vicioso o círculo virtuoso? Recuperado de:

http:// www. pensamientoiberoamericano. org/ xnumeros/ 0/ pdf/ pensamientolbe roamericano- 25 . pdf

Sassen, S. (2007). Nuevas Clases Globales. En Una sociología de la globalización. (pp 205- 233) Buenos Aires, Argentina: Katz.

Segato, R. (2003). Las estructuras elementales de la violencia. Buenos Aires, Argentina: Universidad Nacional del Quilmes.

Stefoni, C. (2009). Inmigrantes en Chile. Una integración diferenciada al mercado laboral. Recuperado de http:// ebookbrowse.com/c-stefoniinmigrantes-en-chile-una-integracion-diferenciada-al-mercado-laboral-pdf$\underline{\text { d353985874 }}$

Stefoni Espinoza, C. (2002). Inmigración peruana en Chile, una oportunidad para la integración. Santiago, Chile: Universitaria.

Triviño Caballero, R. (2012). Mujeres migrantes y misoprostol: aborto privado, escándalo público. Dilemata, 4 (10), 31-44. Disponible en

http:/ / www. dilemata. net/ revista/ index. php/ dilemata/ article/ view/ 169/ 213

Vulpiani, P. (2000). Health for All? Inequalities, Discriminations and Health Care for Migrants. En P. Vulpiani, J.M Comelles y E. Van Dongen, (Eds.) Health for 


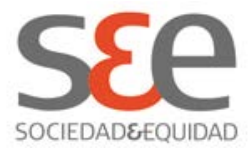

All, All in Health. European Experiences on Health Care for Migrants. (pp 1730): Cidis/ Alisci

Williams, T. Schutt-Ainé, J. y Cuca, Y.(2001). Evaluación de la Calidad de los Servicios de Planificación Familiar a Través de Encuestas de Satisfacción de Clientes. Family planning Perspectives, (Número especial de 2001) ,14-23. Disponible en http:/ / www.guttmacher.org/ pubs/ journals/2701401S. pdf 